\title{
TTC- Based Test as an Efficient Method to Determine Antibiofilm Activity of Silver Nanoparticles
}

\author{
Joanna Chojniak ${ }^{1, *}$, Izabela Biedron ${ }^{1}$, and Grażyna Płaza $^{1}$ \\ ${ }^{1}$ Institute for Ecology of Industrial Areas, Microbiology Department, Kossutha 6, 40-844 Katowice, \\ Poland
}

\begin{abstract}
Among metal nanoparticles, silver nanoparticles are a widely used in various life sectors such as in biomedical applications, air and water purification, food production, cosmetics, garments and in various household products. There are several methods for production of silver nanoparticles. Generally, silver nanoparticles can be prepared by chemical methods such as chemical reduction and electrochemical techniques, physical methods, and biological methods such as the use of microorganisms. The biological route of synthesis provides a great diversity in choice for its raw materials such as bacteria, algae, fungi and plants. The aim of the study was to evaluate the tetrazolium/formazan test as a method to determine antibiofilm activity of biological synthetized silver nanoparticles. In this study Bacillus subtilis grown on brewery effluent and produced biosurfactant was used for silver nanoparticles (Ag-NPs) synthesis. The culture supernatants were used in synthesis of Ag-NPs. The formation of nanoparticles accompanied by colour changes of the used reaction system was confirmed by UV-vis spectroscopy. The bacteria isolated from the biofilm of water supply system were used in the evaluation of the antibiofilm activity of biologically synthetized Ag-NPs. To compare the results the commonly used crystal violet assay (CV) for biofilm analysis was applied.
\end{abstract}

\section{Introduction}

\subsection{Biosynthesis and characterization of silver nanoparticles}

Recently subject of many investigations have become metal nanoparticles. Most frequently used methods of synthesis of metal nanoparticles are chemical and physical methods. In this methods microwaves, ultrasound, irradiation, mechanical grinding are used. Both methods have a negative features, for example, using toxic solvents to the synthesis, during the process of hazardous intermediates are produced, and the processes are often high energy $[1,2]$. The alternative way to the processes is biological synthesis of metal nanoparticles (biosynthesis). In this method microbial activities of bacteria, fungi,

*Corresponding author: chojniak@ietu.katowice.pl 
yeast are used, and plant extract are applied. The synthesis of silver nanoparticles used microbiological method is the important part of nanobiotechnology, connecting the range of biotechnology research with nanotechnology. However, despite some reports, still many aspects of nanoparticles biosynthesis should be studied in detail It was observed that biosurfactants produced by microorganisms play very important role in aggregation and stabilization process [3-13]. They adsorbed onto Ag-NPs surface stabilizing the nanoparticles and prevent their aggregation. Biosurfactants of biological origin have a lot of positive properties: most of them are rapidly degraded, they are not toxic, their activity is in a wide range of temperature, salinity and acidity $[14,15,17-21]$.

\subsection{Biofilm in water drinking distribution}

The drinking water distribution system is the environment where biofilm is formed, regardless of used disinfectants. Control of the development of this structure is important for both aesthetic, health and financial reasons. Formation of biofilm in water distribution system consists of several stages (surface conditioning, irreversible attachment, colonization, detachment) and it is regulated by physical, chemical and biological processes. The first phase is a non-specific and reversible process of binding the bacteria cell to the surface. In the next phase the bacteria secrete the insoluble extracellular polymeric substances (EPS) and form the hydrogel matrix in which cells are embedded. With the increasing amount of EPS a mature biofilm is developing [21, 23]. Bacteria which make up biofilm on the water-pipes' internal surfaces produce great amounts of extracellular polymer substances (EPS). It is a complex mixture of proteins, polysaccharides, lipid acids and nucleic acids. Composition and structure of EPS influences on biofilm integrity, both in terms of physiology and structure as well as have direct impact on physical and chemical properties as well as biological properties of biofilm. Polymer substances essentially improve adhesion as well as make up a barrier which protects bacteria cells from the impact of adverse factors such as disinfecting agents [24]. Microorganisms resistant to antibiotics in water distribution system also exhibit resistance to chlorination. Environmental stress caused by the disinfection process may consequently increase the resistance to antibiotics [25]. Biofilm forming microorganisms are far more resistant to environmental stress, thus the first selection emerged microorganism whose high ability for adhesion and simultaneous resistance to antibiotics, which confirms the direct descent of biofilm. In earlier studies were selected 11 strains accepted as a model group for biofilm formation in water distribution system. Selection of microorganisms based on the identification of high ability to adhere and presenting resistance against antibiotics and chemotherapeutics. Ability to adhesion is a source of information on the actual response of the microorganism to conditions in the environment.

The aim of the study was to evaluate the impact of biological synthetized Ag-NPs on the biofilm formation by the TTC method. Also, the CV assay was used to compare the results.

\section{Materials and Methods}

\subsection{Biosynthesis and characterization of silver nanoparticles}

Bacillus subtilis isolated form the petroleum contaminated soil was used in the synthesis of Ag-NPs [26]. The strain was identified and characterized in earlier studies [27]. The strain was grew on the brewery wastewater at $30^{\circ} \mathrm{C}$ for 96 hours, under shaking. After incubation the culture was centrifuged by $10 \mathrm{~min}, 5000 \mathrm{rpm}$. The free-cell supernatant was 
used for biosynthesis of $\mathrm{Ag}-\mathrm{NPs} . \mathrm{AgNO}_{3}$ was added to the supernatant in final concentration $1 \mathrm{mM}$. The solution was incubated at $30^{\circ} \mathrm{C}$ for 48 hours. The presence of nanoparticles was monitored by UV-Vis spectrophotometer at a wavelength of 300-700 nm. The concentration of Ag-NPs measured by atomic absorption spectroscopy was around $165 \mathrm{mg} / \mathrm{L}$.

\subsection{Bacterial strains}

Biofilm samples were collected from drinking water distribution system in Wroclaw. Morphological characterization (colony colour, shape and size) was recorded on selective media and biochemical characterization was tested according to API Zym test [28]. The identification of selected strains was carried out by the sequencing and comparative analysis of $16 \mathrm{~S}$ rDNA,. The isolates were detected representatives of the genus: Advanella Microbacterium, Kocuria, Moraxella, Enhydrobacter, Micrococcus, Pseudomonas, Bacillus, Psychrobacter. Selected strains were grown on LB agar plates at $22^{\circ} \mathrm{C}$. The single isolated colony was inoculated in sterile LB broth and incubated at $22^{\circ} \mathrm{C}$ in rotary shaker (150 rpm) for 24-48 hours. After incubation, culture was centrifuged at $5000 \mathrm{rpm}$ for 10 minutes.

\subsection{Evaluation of Ag-NPs impact on the biofilm formation by selected bacteria}

\subsubsection{Crystal violet assay}

The bacterial suspension was adjusted to $0.5 \mathrm{McF}$ arland's standard. A volume of $100 \mu 1$ of bacterial suspension was added to each well on microtiter plate. The silver nanoparticles were added to the suspensions and incubated $48 \mathrm{~h}$ at $22^{\circ} \mathrm{C}$. After incubation the medium was discarded from the each well and thoroughly washed with PBS (Phosphate-buffered saline). A volume of $100 \mu \mathrm{l}$ of $0.1 \%$ crystal violet was added to each well and left for $30 \mathrm{~min}$ at room temperature. The stain was discarded and plate was thoroughly washed by PBS. The remaining in biofilm structure stain was solubilized with $200 \mu 1$ of $95 \%$ ethanol. The concentration of $\mathrm{CV}$ was determined by measuring the optical density of solution at $620 \mathrm{~nm}[15]$.

\subsubsection{TTC assay}

The bacterial suspension was adjusted to 0.5 McFarland's standard. A volume of $100 \mu 1$ of bacterial suspension was added to each well on microtiter plate. A volume of $10 \mu \mathrm{l}$ of silver nanoparticles solution was added to the suspensions and incubated 24 hours and 72 hours at $22^{\circ} \mathrm{C}$. After incubation, the medium was discarded from every well and thoroughly washed twice with PBS to remove unbound cells. A volume of $100 \mu 1$ of $0.01 \%$ solution of TTC was added to each well and incubated for 24 hours at $22^{\circ} \mathrm{C}$. After incubation, TTC solution was removed and microplate was air dried. Bound red formazan was dissolved using $20 \%$ acetone and $80 \%$ ethanol. The concentration of reduced TTC was determined by measuring the optical density of solution at $500 \mathrm{~nm}$ [19]. Each experiment was done in 3 replicates. Cell-free supernatant contained only biosurfactant and AgNPs + biosurfactant solution were used as the controls. The impact AgNPs was detected in indirect way, eg.

$$
\mathrm{OD}_{\mathrm{AgNPs}}=\mathrm{OD}_{\mathrm{AgNPs}+\text { biosurfactant solution }}-\mathrm{OD}_{\text {cell-free supernatant }}
$$


Also, the bacterial cultures were used to evaluate the biofilm formation.

\section{Results and Discussion}

During the synthesis of silver nanoparticles the colour of the solution was changed. The presence of silver nanoparticles was monitored by UV-Vis spectrophotometer in the range of 300-700 nm (Fig. 1). The typical peak between 400-410 nm for Ag-NPs was observed.

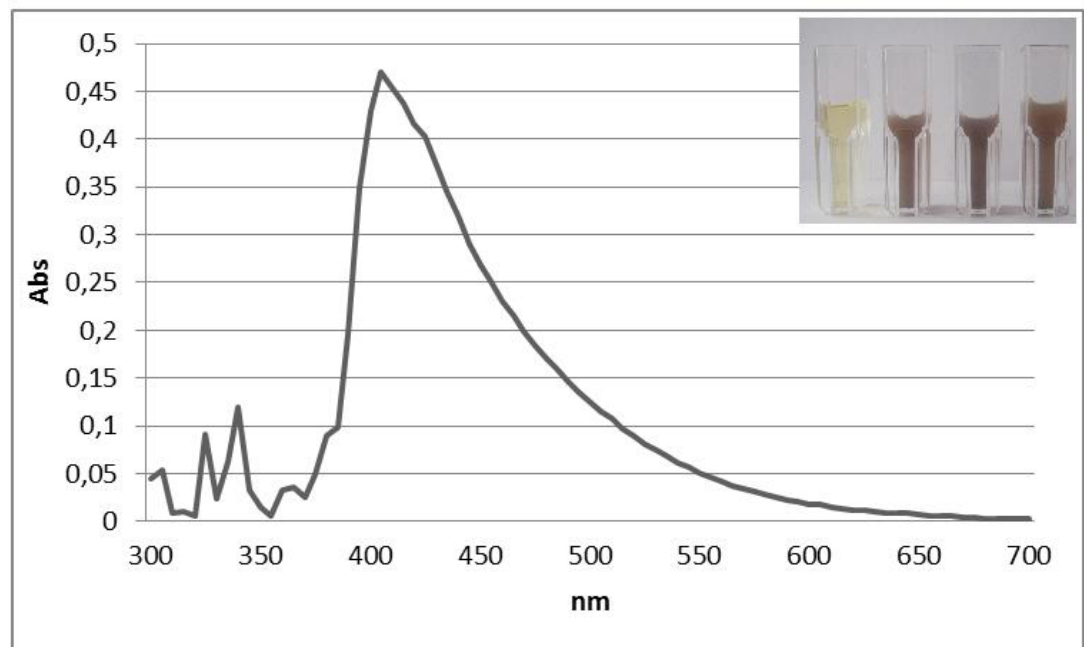

Fig. 1. Graph with absorption spectrum from UV-Vis spectrophotometer and the colour of solution.

Crystal violet (CV) staining was used as colorimetric method to measure biofilm formation in the presence of silver nanoparticles [16]. Based on the results obtained a greater inhibition of the biofilm formation was observed in the solution containing silver nanoparticles and biosurfactant. Nine strains showed a reduced level of adhesion after 24 hours of incubation as a result of silver nanoparticles solution (Fig. 2). In the case of two strains: Bacillus thuringiensis (5) and Moraxella osloensis or Enhydrobacter aerosaccus (2), where there was no observed the reduction in biofilm formation. The strains are Gram positive and produce significant amount of EPS composed mainly from proteins [28]. Also strain belong to the genus Microccocus (10) showed no significant decrease of biofilm formation. The largest adhesion inhibition was observed in two strains: Advenella incenata (6) $-65,31 \%$ and strain belong to the genus Microccocus (9)- 80,92\%. After 72 hours of incubation, 10 of 11 strains showed $40 \%$ or more reduction in adhesion as a result of silver nanoparticles. The highest inhibition of biofilm formation was observed, for Advenella incenata (1), 86.85\% (Fig. 3). It was a strain which showed very high antibiotic resistance [28]. No impact of silver nanoparticles was observed for strain 3-Kocuria rhizophila or Psychrobacter sp. 


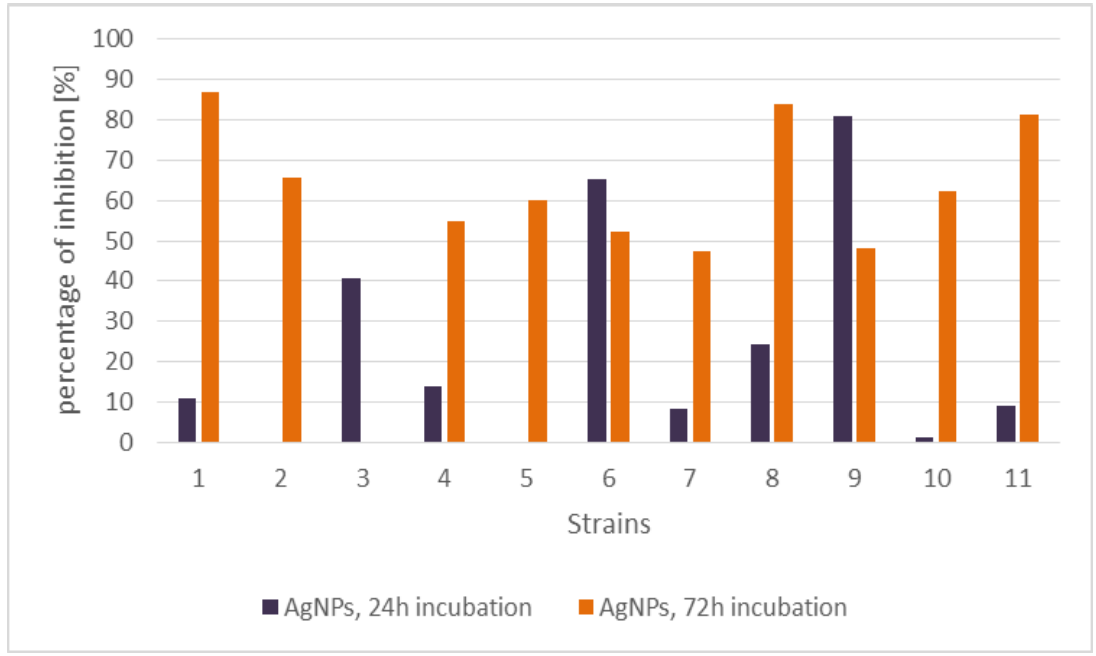

Fig. 2. Percentage of inhibition formation biofilm in Crystal Violet (CV) method. Strains: 1. Advenella incenata, 2. Moraxella osloensis or Enhydrobacter aerosaccus, 3. Kocuria rhizophila or Psychrobacter sp., 4. Microbacterium oxydans, 5. Bacillus thuringiensis, 6. Advenella incenata, 7. genus Advenella, 8. Micrococcus luteus, 9. genus Micrococcus, 10. genus Micrococcus, 11. genus Pseudomonas.

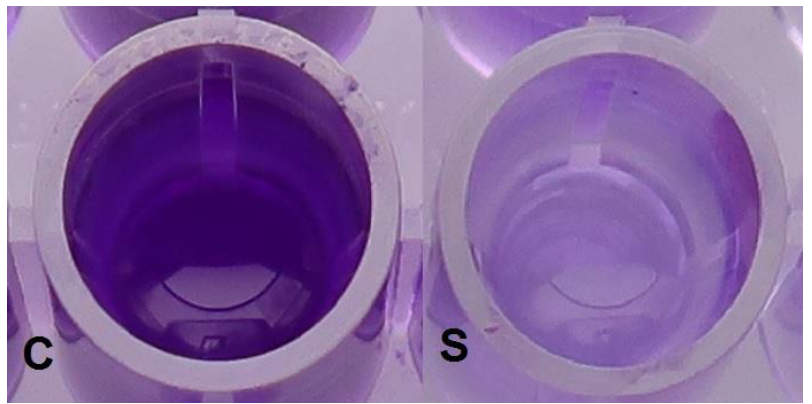

Fig. 3. The highest inhibition of biofilm formation in Advenella incenata (1) (C- control, S- sample).

The TTC assay is quantification techniques based on the metabolic activity of biofilm cells. In this method tetrazolium salt is converted by metabolically active cells to a colored formazan. The TTC assay results indicate minimum inhibition of dehydrogenase activity of biofilm. Significantly inhibition was observed in Microbacterium oxydans (4) after $72 \mathrm{~h}$ of incubation. There was no impact of nanoparticles for dehydrogenase activity in strain (1) Advenella incenata, wherein the highest inhibition of biofilm formation was observed in CV assay. 


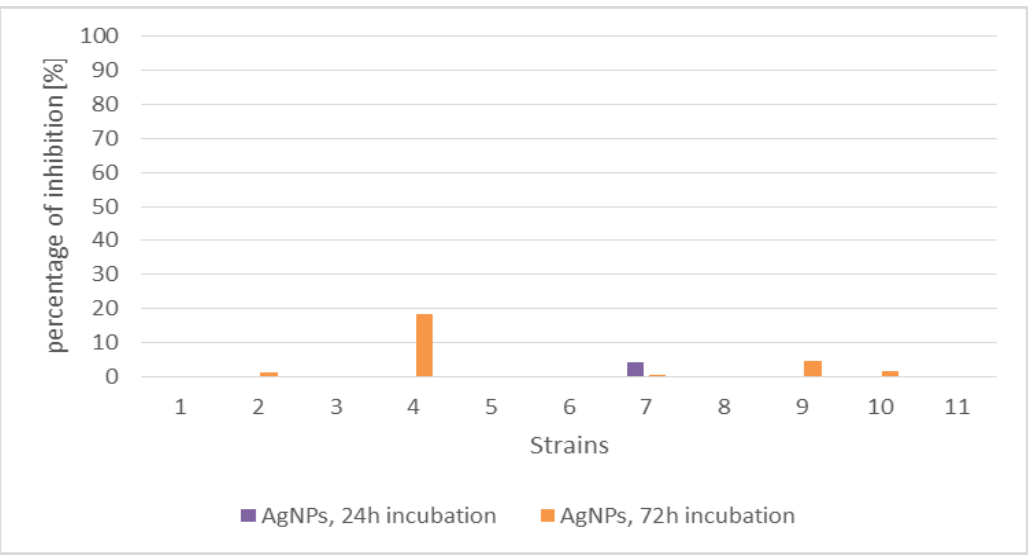

Fig. 4. Percentage of inhibition cellular respiration in TTC method. Strains: 1. Advenella incenata, 2. Moraxella osloensis or Enhydrobacter aerosaccus, 3. Kocuria rhizophila or Psychrobacter sp., 4. Microbacterium oxydans, 5. Bacillus thuringiensis, 6. Advenella incenata, 7. genus Advenella, 8. Micrococcus luteus, 9. genus Micrococcus, 10. genus Micrococcus, 11. genus Pseudomonas.

From last few years studies of AgNPs as antibacterialn and antibiofilm agents have increased. Nano-sized silver particles can be prevalent for much longer time than silver ions and act on sites within bacterial cells that are critical to physiological function (e.g. cell wall, DNA/RNA, protein syntheses and electron transport). It is said that the effect of AgNPs on biofilm removal was size-dependent, smaller nanoparticles had the higher impact on biofilm structure [29]. In our study we tested nanoparticles on environmental strains, in contrast to research on the model strains. Perez-Diaz et al. and Martinez - Gutierrez observed antibiofilm and antibacterial properties of AgNPs [30, 31]. We confirmed activity of AgNPs as anti-biofilm agent, but we weren't observed decrease in respiratory activity by TTC assay, suggesting biocidal properties. Due to the non-specific efficient of AgNPs we think that nanoparticles have especially influence to metabolic reactions that controls the production of EPS compounds [31]. We have obtained results indicating that nanoparticles inhibited biofilm production by blocking the formation of exopolysacharides, which allows us to claim that to assessment antibiofilm properties, CV assay is more appropriate.

\section{Conclusions}

1. Probably, the silver nanoparticles inhibited biofilm production by blocking the formation of EPS. Therefore the crystal violet method is more reliable in determining of the biofilm inhibition than TTC assay.

2. Each strain has its own resistance against the silver nanoparticles.

3. The results of silver nanoparticles activity were observed after prolonger incubation.

The study was supported by the National Science Centre (Poland) (Project No. 2013/09/B/NZ9/01759 (decision no. 2013/09/B/NZ9/01759)

\section{References}

1. K. Patel, S. Kapoor, D.P. Dave, T. Ukherjee, J. Chem. Sci. 117, 311 (2007)

2. P. Sileikaite, I. Prosycevas, J. Puiso, A. Juraitis, A. Guobiene, Mater Sci. 12, 287 (2006) 
3. A.K. Mittal, Y. Chisti, U.C. Banerjee, Biotechnol. Adv. 31, 346 (2013)

4. A.S. Reddy, C.Y. Chen, C.C. Chen, J.S. Jean, Fan C.W., H.R. Chen, J.C. Wang, V.R. Nimje, J.Nanosc.Nanotech. 9, 6693 (2009b)

5. A.S. Reddy, C.Y. Chen, S.C. Baker, C.C. Chen, J.C. Jean, C.W. Fan, H.R. Chen, J.C. Wang, Mat. Letters 63, 1227 (2009a).

6. B.R. Singh, S. Dwivedi, A.A. Al-Khedhairy, J. Musarrat, Coll.Surf.B: Bioint. 85, 207 (2011)

7. C. Tamuly, M. Hazarika, S.Ch. Borah, M.R. Das, M.P. Boruah, Coll. Sur.B: Biointerfaces 102, 627 (2013)

8. G.G. Kumar, S.K. Mamidyala, Coll.Sur.B: Bioint. 84, 462 (2011)

9. G.S. Kiran, A. Sabu, J. Selvin, J. Biotechnol. 148, 221 (2010)

10. G.V. Kumar, D.S. Gokavarapu, A. Rajeswari, S.T. Dhas, V. Karthick, Z. Kapadia, T. Shrestho, A.I. Barathy, A. Roy, S. Sinna, Colloids Surfaces B. Biointerface. 87, 159 (2011)

11. I.M. Banat, A. Franzetti, I. Gandolfi, M.G. Martinotti, L. Fracchia, T.J. Smyth, R. Marchant, Microbial Biosurfactants Production, Applications And Future Potential, Appl. Microbiol. Biotechnol., 87, 427 (2010)

12. X. Zhang, S. Yan, R.D. Tyagi, R.Y. Surampalli, Chemosphere 82, 489 (2011)

13. Y.J. Somg, K-H Jang, S.B. Kim, Progress Biochemistry. 44, 1133 (2009)

14. D. Bhattacharya, R.K. Gupta, Crit. Rev. Biotechnol. 25, 199 (2005)

15. D.K. Bozanic, S. Dimitrijevic- Brankovic, N. Bibic, A.S. Luyt , V. Djokovic, Morphology, Optical And Antimicrobial Properties. Carbohyd. Polymers, 83, 883 (2011).

16. D. Dusane, V. Pawar, Y. Nancharaiah, V. Venugopalan, A. Kumar, S. Zinjarde, Biofouling, vol. 27, 645 (2011)

17. K.B. Narayanan, N. Sakthivel, Add. Coll. Int. Sc. 156, 1 (2010)

18. L. Kvitek, A. Panacek, J. Soukupova, M. Kolar, R. Vecerova, R. Prucek, M. Holecova, R. Zboril, J. Phys. Chem. C 112, 5825 (2008)

19. M. Gericke, A. Pinches, Hydrometallurgy, 83, 132 (2006)

20. P.S. Chandran, M. Chaudhary, R. Pasricha, A. Ahmad, M. Sastry, Biotechnol. Prog. 22, 277 (2006)

21. T. Leung, Ch.K. Wong, Y. Xie, Mat. Chem.Physics, 121, 402 (2010)

22. L. F. Melo, F. J. Mergul, vol. 2011, 1414 (2014)

23. M. J. Lehtola, I. T. Miettinen, M. M. Keinänen, T. K. Kekki, O. Laine, A. Hirvonen, T. Vartiainen, and P. J. Martikainen, Water Res., vol. 38, no. 17, 3769 (2004)

24. Z. Xue, V. R. Sendamangalam, C. L. Gruden, and Y. Seo, Environ. Sci. Technol., vol. 46, 13212 (2012)

25. C. Xi, Y. Zhang, C. F. Marrs, W. Ye, B. Foxman, J. Nriagu, C. Xi, Y. Zhang, C. F. Marrs, W. Ye, C. Simon, B. Foxman, and J. Nriagu, Appl. Environ. Microbiol. Vol. 75 nr. 17, 5714 (2009)

26. C.J. Berry, S. Story, D.J. Altman, R. Upchurch, W. Whitman, D. Singleton, G. Płaza, R.L. Brigmon, Biological Treatment of Petroleum and Radiological Contaminated Soil (Oxford University Press., 2006) 
27. G.A. Płaza, M. Pacwa - Płóciniczak, Z. Piotrowska - Seget, R. Brigmon, E. Król, Characterization of Bacillus strains producing biosurfactants (Springer Science Business Media, 2015)

28. I. Biedroń, T. Traczewska, G. Płaza, Rocz. Ochr. Śr.,18(2), 86 (2016)

29. C.Y. Loo, P.M. Young, R. Cavaliere, C.B. Whitchurch, W.H. Lee, R. Rohanizadeh, Drug Dev Ind Pharm. 40(6), 719 (2014)

30. F. Martinez-Gutierrez, L. Boegli, A. Agostinho, E.M. Sánchez, H. Bach, F. Ruiz, G. James, Biofouling. 29(June), 651 (2013)

31. M.A. Pérez-Díaz, L. Boegli, G. James, C. Velasquillo, R. Sánchez-Sánchez, R.E. Martínez-Martínez, G.A. Martínez-Castañón, Martinez-F. Gutierrez, Mater Sci Eng C., 55, 360 (2015) 